\title{
HIGHER HOMOTOPY-COMMUTATIVITY
}

\author{
BY
}

FRANCIS D. WILLIAMS $\left({ }^{1}\right)$

Introduction. In topology, there is a significant class of $H$-spaces which are homotopy-commutative, but not strictly commutative. As examples, one has the loop spaces of $H$-spaces, for which, in general, no strictly commutative form exists. However, these loop spaces are known, cf. [15], to satisfy far stronger homotopycommutativity properties than the usual one. It is the purpose of this paper to introduce a new sequence of strong forms of homotopy-commutativity, called $C_{n}$-forms, which are possessed by these loop spaces, and to examine the geometric implications of a space possessing such a form.

The classical Hopf construction characterizes the $H$-spaces as being those spaces which can be the null-homotopic fiber of a fibration over their own suspension. Further, it follows from work of Stasheff, [11], or by a direct construction of the present author, [17], that among the homotopy-associative $H$-spaces, the homotopycommutative ones are those for which the Hopf construction extends to a fibration over the James' two-fold reduced product space of their suspension. Now spaces which are fibered over $n$-fold reduced product spaces are important; they have been used, for example, in the computation of homotopy groups, cf. Toda, p. 174 of [16]. It will be the principal result of this paper that those topological monoids for which the Hopf construction extends to a fibration over the $n$-fold reduced product space of their suspension are exactly those which admit $C_{n}$-forms. (Although it is possible to define the concept of $C_{n}$-form in the category of Stasheff's $A_{n+1}$-spaces, the overwhelming bulk of notation required in that case causes the present paper to deal with only the strictly associative case.)

The morphisms in the category of spaces with $C_{n}$-forms will be introduced, and these morphisms will be used to determine when certain induced fiber spaces admit $C_{n}$-forms. This, in turn, leads to a characterization in terms of $k$-invariants of certain spaces which admit $C_{n}$-forms.

The organization of this paper is as follows. The first section gives general background material and definitions. The concept of $C_{n}$-form is introduced in an intuitive fashion, the basic results of the paper are discussed informally, and indications are given as to future research possibilities in this area. The second section presents the precise definitions of the $C_{n}$-forms, states the main theorems, and gives applications. The proofs are contained in the third section.

Received by the editors March 1, 1968 and, in revised form, June 19, 1968.

(1) Supported by the National Science Foundation Grant GP-6318. 
The bulk of the material in this article was contained in the author's Ph.D. thesis at the University of Wisconsin under the supervision of S. Y. Husseini, whose interest, encouragement, and many suggestions were invaluable.

1. The concept of $H$-space is one which has been the subject of much research in recent years. (Recall that a topological space is called an $H$-space if there is a continuous map $m: X \times X \rightarrow X$, written $m(x, y)=x y$, and an element $e$ in $X$ such that $e x=x=x e$ for every $x$ in $X$.) The importance of $H$-spaces has come about both because $H$-spaces arise in many more varied contexts than their parents, the topological groups, and because many of the results of the theory of topological groups remain valid in the more general context of $H$-spaces.

In many situations, the notions of associativity and commutativity of the multiplication in an $H$-space are too strict. In the case of associativity, the weaker property of homotopy-associativity (i.e., $(x y) z$ is homotopic to $x(y z)$ ) occurs more frequently in practice. Similarly, homotopy-commutativity ( $x y$ homotopic to $y x$ ) replaces strict commutativity in many cases. As was mentioned in the Introduction, a well-known example of this phenomenon occurs in the space of loops on an $H$-space.

One of the principal methods used in the investigation of $H$-spaces has been the interplay between their algebraic properties, such as those discussed in the preceding paragraph, and certain geometric properties. The first results of this nature were in the development of the Hopf construction, due to H. Hopf [4], and later extended by Spanier-Whitehead [10] and Sugawara [13]. This construction characterizes a space as being an $H$-space if and only if it can appear as the fiber in a certain type of fibration. The Hopf construction will be reviewed in \$3. In [14], Sugawara extended this method to characterize homotopy-associative $H$-spaces, and the author, in [17], has given the analogous characterization of $H$-spaces which are both homotopy-associative and homotopy-commutative. Perhaps the most spectacular results in this line are those of Stasheff's theory of $A_{n}$-spaces [14], in which he determines homotopy-associativity criteria for deciding which $H$-spaces admit classifying spaces.

In discussing the two permutations of the product of two elements of an $H$-space, $X$, there is no need to worry about associativity of the product. However, when one begins to consider the various permutations of products of more than two elements of $X$, the problem of nonassociativity rears its ugly head. To dismiss this problem once and for all, the forms of higher homotopy-commutativity considered herein will be defined only for associative $H$-spaces, called topological monoids. In dealing with loop spaces, this is no real restriction since there is a well-known associative model of the space of loops, given as follows: Let $X$ be a based space with base point $x_{0}$. Then the Moore space of paths in $X, P X$, consists of all pairs $(\lambda, r)$, where $\lambda:[0, r] \rightarrow X$ and $\lambda(0)=x_{0}$. The Moore loop space of $X, \Omega X$, consists of all $(\lambda, r)$ in $P X$ for which $\lambda(r)=x_{0}$. The pair $(\lambda, r)$ will be denoted by $\lambda^{r}$. 
Sugawara [15] characterized the loop space of an $H$-space by strengthened homotopy-commutativity designed to insure the existence of $H$-structure in its classifying space. These conditions are described in Definition 7 of $\$ 2$ of this paper. It is not known what the implications of Sugawara's conditions are in the context of the Hopf-type constructions.

Before discussing the $C_{n}$-forms of homotopy-commutativity, we introduce some notation and terminology. All spaces, unless otherwise specified, will be assumed to have base points. In the case of an $H$-space, the base point will be taken to be the identity element, and in the case of a $\mathrm{CW}$-complex, the base point will be assumed to be a 0 -cell. The suspension of a space $X$, denoted by $S X$, will be the product of $I$ (the unit interval) with $X$, with the subspace $(\partial I \times X) \cup\left(I \times\left\{x_{0}\right\}\right)$ identified to a point. The $n$-fold Cartesian product of $X$ with itself will be denoted by $X^{n}$. We are now prepared to discuss the higher forms of homotopy-commutativity.

The basic idea is simple and intuitive. A homotopy-commutativity is simply a homotopy connecting $x y$ with $y x$, as described above. Now if a monoid, $X$, is homotopy-commutative, then a chain connecting the various permutations of a product of three elements of $X$ is given as follows:

$$
x y z \sim x z y \sim z x y \sim z y x \sim y z x \sim y x z \sim x y z .
$$

The pattern here is that two permutations are connected by a homotopy which commutes adjacent elements. Thus a map is defined $\partial I^{2} \times X^{3} \rightarrow X$. If this map extends to a map $I^{2} \times X^{3} \rightarrow X$, then $X$ satisfies the next higher degree of homotopycommutativity. Ordinary homotopy-commutativity will be called a $C_{2}$-form, and this new map, if it exists, will be called a $C_{3}$-form on $X$, where the subscripts refer to the number of elements of $X$ being permuted. Now if such a $C_{3}$-form exists, then using it a map may be defined from $\partial I^{3} \times X^{4} \rightarrow X$. If this extends to a new map $I^{3} \times X^{4} \rightarrow X$, then $X$ admits the next higher form of homotopy-commutativity, and this map is called a $C_{4}$-form, and so on. In general, if a space admits $C_{i}$-forms, $2 \leqq i \leqq n-1$, then a map is defined from $\partial I^{n-1} \times X^{n} \rightarrow X$, by using these forms, which connects all the permutations of a product of $n$ elements of $X$. If this map extends to $I^{n-1} \times X^{n} \rightarrow X$, this extension will be called a $C_{n}$-form. It takes some care to divide up properly the boundary of $I^{n}$ so that the permutations will be connected in the right way. Although the basic idea is simple, the notation is somewhat complicated. These concepts occupy Definition 1, 2, 4, and 5 of $\$ 2$.

In this paper, we exploit the $C_{n}$-forms to obtain theorems of the Hopf construction type which characterize monoids which admit them, c.f. Theorem 14. Further, obstruction theoretic and dimensionality arguments are used to obtain some technical results about spaces admitting $C_{n}$-forms, which are then applied to give a characterization of $C_{n}$-spaces in terms of their $k$-invariants.

Several areas of investigation on $C_{n}$-spaces lie ahead. In a future paper, the author will describe the relationship between the $C_{n}$ notion of homotopy-commutativity and the generalized higher Whitehead products of Porter, [9]. We also 
intend to develop the homology analogue of $C_{n}$-commutativity so that more effectively computable invariants of $C_{n}$-spaces may be obtained.

2. In this section the precise definition of $C_{n}$-forms will be given and the main theorems about them will be stated. The first step will be to determine how to arrange the various permutations of a product of $n$ elements of a monoid around the boundary of an $(n-1)$-cell. This motivates the following definitions.

Definition 1. Let $\boldsymbol{n}$ denote the sequence $(1,2, \ldots, n)$. Subsequences of $\boldsymbol{n}$ will be denoted by symbols $A_{l}, B_{m}$, etc., where the subscripts denote the number of elements in the subsequence. The function $i_{A}: A_{l} \rightarrow n$ will be the inclusion, and $A$ will denote the composition $l \rightarrow A_{l} \rightarrow \boldsymbol{n}$. An ordered pair, $\left(A_{l}, B_{m}\right)$, of disjoint subsequences of $\boldsymbol{n}$ is called a $(l, m)$ partition, or shuffle, of $\boldsymbol{n}$ if $\operatorname{Im}\left(i_{A}\right) \cup \operatorname{Im}\left(i_{B}\right)=\boldsymbol{n}$. Analogously, an ordered $k$-tuple $\left(A_{l_{1}}, \ldots, A_{l_{k}}\right)$ of disjoint subsequences of $n$ is an $\left(l_{1}, \ldots, l_{k}\right)$ partition if their union is equal to $n$. We shall utilize the following description of a cell-complex structure on the $(n-1)$-cell which is due to Milgram, [Definition 4.1 of 8], who observed its importance in connection with interated loop spaces.

Definition 2. Consider $n=(1,2, \ldots, n)$ as a point in $R^{n}$. The symmetric group $S_{n}$ acts on $R^{n}$ by permuting the coordinates. Let $K_{n}$ be defined to be the convex hull of the orbit of $\boldsymbol{n}$ under this action.

From this definition, it is immediate that $K_{n}$ is homeomorphic to an $(n-1)$ dimensional cell. The next two theorems are Lemmas 4.2 and 4.3 of [8].

THEOREM 3. The cells, $K_{n}$, satisfy the following properties.

1. The boundary of $K_{n}, L_{n}$, is the union of $(n-2)$-cells which are in one-to-one correspondence with the 2-fold partitions of $n$.

2. If $\left(A_{l}, B_{m}\right)$ is such a partition, then the cell of $L_{n}$ corresponding to it is the image of $K_{l} \times K_{m}$ by

$$
V\left(A_{l}, B_{m}\right): K_{l} \times K_{m} \rightarrow L_{n},
$$

a linear homeomorphism into $L_{n}$. $\left(V\left(A_{l}, B_{m}\right)\left[K_{l} \times K_{m}\right]\right.$ will be denoted by $K_{l}^{A} \times K_{m}^{B}$. $)$

3. Two such cells of $L_{n}$, say $K_{l}^{A} \times K_{m}^{B}$ and $K_{r}^{A^{\prime}} \times K_{s}^{B^{\prime}}$, intersect if and only if there are partitions $\left(C_{p}, D_{q}\right)$ of $l$ and $\left(C_{j}^{\prime}, D_{k}^{\prime}\right)$ of $s$ such that $(p, q, m)=(r, j, k)$, and the diagram

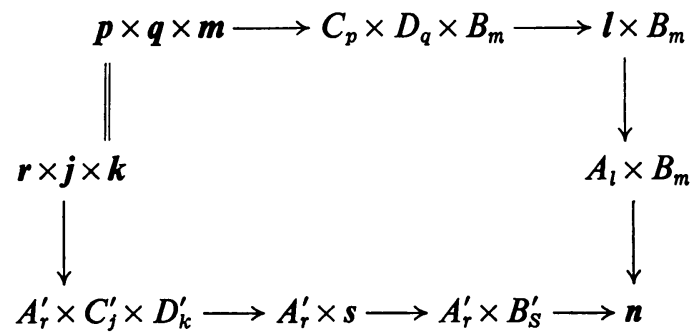

is commutative. In this case, their intersection is given by the commutativity of the following diagram. 


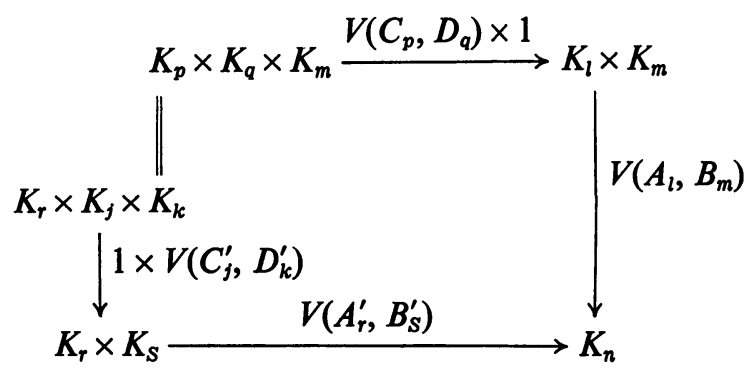

The cell complexes $K_{i}$ admit more structure, as given in the following lemma. The maps guaranteed by this lemma may be thought of as degeneracy operators.

Lemma 4. There are maps $s_{j}: K_{n+1} \rightarrow K_{n}, j=1, \ldots, n+1$, such that (1) if $j=A(p)$, then the diagram

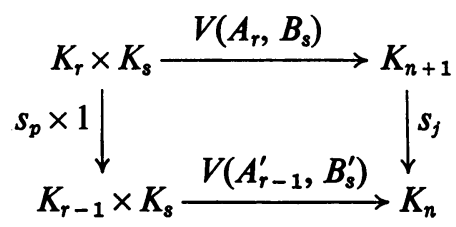

is commutative, where $\left(A_{r-1}^{\prime}, B_{s}^{\prime}\right)$ is the partition of $n$ given by
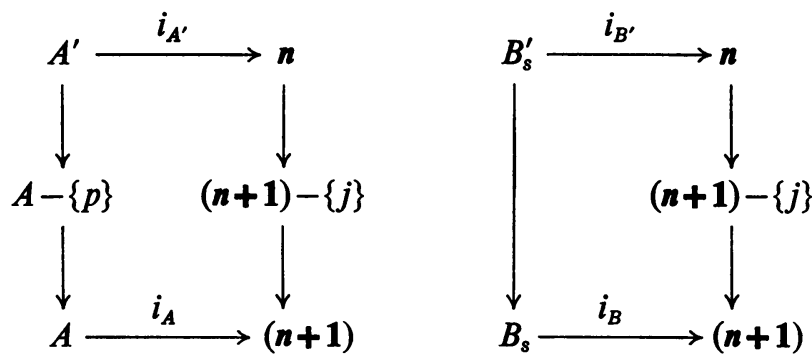

where the unlabelled arrows are injections;

(2) if $j=B(q)$, then the diagram

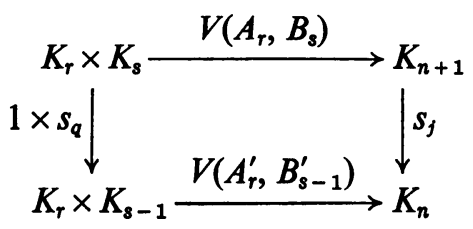

is commutative, where $\left(A_{r}^{\prime}, B_{s-1}^{\prime}\right)$ is defined in similar fashion to $\left(A_{r-1}^{\prime}, B_{s}^{\prime}\right)$ in (1) and 
(3) if $i<j$, then

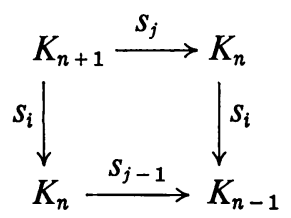

is commutative.

It is now possible, using this terminology, to make precise the intuitive definition of the higher homotopy-commutativity which was discussed above. The following is the basic definition.

Definition 5. Let $X$ be a topological monoid and $n \geqq 1$. Then a $C_{n}$-form $\left\{Q_{i}\right\}$, $i=1, \ldots, n$, on $X$ is a set of maps $Q_{i}: K_{i} \times X^{i} \rightarrow X$ such that

(1) $Q_{1}: K_{1} \times X \rightarrow X$ is the identity map of $X$,

(2) $Q_{i}\left(V\left(A_{r}, B_{s}\right)[\rho, \sigma], x_{1}, \ldots, x_{i}\right)=Q_{r}\left(\rho, x_{A(1)}, \ldots, x_{A(r)}\right) \cdot Q_{s}\left(\sigma, x_{B(1)}, \ldots, x_{B(s)}\right)$, where $\rho \in K_{r}, \sigma \in K_{s}$, and $x_{1}, \ldots, x_{i} \in X$, and

(3) if $x_{j}=e$, the identity of $X$, then

$$
Q_{i}\left(\tau, x_{1}, \ldots, x_{i}\right)=Q_{i-1}\left(s_{j}(\tau), x_{1}, \ldots, \hat{x}_{j}, \ldots, x_{i}\right)
$$

A space, $X$, together with a $C_{n}$-form on $X$, is called a $C_{n}$-space. It may happen that a space admits maps $\left\{Q_{i}\right\}$ for all $i \geqq 1$, such that $\left\{Q_{i}\right\}, i=1, \ldots, n$, is a $C_{n}$-form for every $n$. In this case $X$ is called a $C_{\infty}$-space.

REMARK 6. The fact that the definitions of the $Q_{i}$ are consistent comes from the following observations.

(1) If two cells of the boundary of $K_{i}$ intersect, the consistency of the definition of $Q_{i}$ along their intersection is verified by part (3) of Theorem 3 .

(2) The consistency of conditions (2) and (3) with each other is verified by parts (1) and (2) of Lemma 4.

(3) The consistency of condition (3) of the definition is given by conclusion (3) of Lemma 4.

EXAMPLeS.

(1) Any commutative topological monoid, $X$, is a $C_{\infty}$-space, by setting

$$
Q_{n}\left(T, x_{1}, \ldots, x_{n}\right)=x_{1} \cdots x_{n}
$$

for all $T$ in $K_{n}, x_{1}, \ldots, x_{n}$ in $X$.

(2) A $C_{2}$-form is simply a commuting homotopy, so the $C_{2}$-spaces are the homotopy-commutative monoids.

(3) If $Y$ is an $H$-space, and $X=\Omega Y$, the associative loop space of $Y$, then $X$ is a $C_{\infty}$-space. Although this fact follows from Example 4, a direct proof will be given in $\$ 3$. 
Before giving Example 4, we introduce some terminology which will be used later to determine the invariants provided by the $C_{n}$-forms. The next definition is due to Sugawara [15].

Definition 7. Let $Y$ and $Z$ be monoids. Then a map $f: Y \rightarrow Z$ is called strongly homotopy-multiplicative if there exist maps

$$
M_{n}: Y \times(I \times Y)^{n} \rightarrow Z, \quad n=0,1, \ldots,
$$

such that

(1) $M_{0}=f$ and

$$
\begin{array}{ll}
M_{n}\left(y_{0}, t_{1}, y_{1}, \ldots, t_{n}, y_{n}\right) & \\
\quad=M_{n-1}\left(y_{0}, t_{1}, y_{1}, \ldots, t_{i-1}, y_{i-1} y_{i}, t_{i+1}, y_{i+1}, \ldots, t_{n}, y_{n}\right) & \text { if } t_{i}=0, \\
\quad=M_{i-1}\left(y_{0}, t_{1}, y_{1}, \ldots, t_{i-1}, y_{i-1}\right) M_{n-i}\left(y_{i}, t_{i+1}, \ldots, t_{n}, y_{n}\right) & \text { if } t_{i}=1 .
\end{array}
$$

We specialize this definition to call $f m$-homotopy-multiplicative if the $M_{n}$ exist for $0 \leqq n \leqq m$. If $X$ is a topological monoid, $X$ will be called strongly homotopycommutative in the sense of Sugawara, if the multiplication $m: X \times X \rightarrow X$ is a strongly homotopy-multiplicative map.

EXAMPLE 4. If $X$ is a monoid which is strongly homotopy-commutative in the sense of Sugawara, then $X$ is a $C_{\infty}$-space. It was pointed out to me by J. D. Stasheff that this fact follows from triangulating the $K_{n}$ 's and setting sufficiently many coordinates equal to the basepoint in Sugawara's definition to obtain maps $\Delta_{n} \times X^{n} \rightarrow X$, then piecing these together. Since this example is essentially equivalent to Example 3, no further details will be given concerning it.

The category of countable $\mathrm{CW}$-complexes which are monoids, and $n$-homotopymultiplicative maps is the true domain of the $C_{n}$-spaces, in the sense indicated by the following two results, whose proofs will be given in $\S 3$.

Proposition 8. Let $Y$ and $Z$ be monoids, where $Y$ is countable $C W$-complex, and $Z$ is a $C_{n}$-space. Let $f: Y \rightarrow Z$ be an $n$-homotopy-multiplicative map which is a weak homotopy equivalence (i.e., $f_{*}: \pi_{*}(Y) \rightarrow \pi_{*}(Z)$ is an isomorphism). Then $Y$ may be given a $C_{n}$-form.

From this proposition follows immediately the following invariance theorem.

THEOREM 9. In the category of countable $C W$-monoids, the property of being a $C_{n}$-space is an invariant of n-homotopy-multiplicative homotopy type.

REMARK 10. Let $(K, L)$ be a pair of complexes. Then $L$ is said to be retractile in $K$, according to [5], provided $N$ is contractible in $M$, where $(M, N)$ is the identification space $(C K, K) /(L, L)$. The following facts about retractile subspaces will be applied to the $C_{n}$-theory.

(1) Let $X$ be an $H$-space. If $L$ is retractile in $K$, then given $f: C Y \times K \rightarrow X$ and $h: C Y \times L \rightarrow X$ such that $f|Y \times L=h| Y \times L$, then $f \mid Y \times K$ extends to $f: C Y \times K \rightarrow X$ such that $f \mid C Y \times L=h$.

(2) The subspace $X^{[i]}$ is retractile in $X^{i}$. 
It follows from these facts that if $\left\{Q_{i}\right\}, i=1, \ldots, n-1$, is a $C_{n-1}$-form on a countable CW-monoid, $X$, and if $Q_{n}^{\prime}: K_{n} \times X^{n} \rightarrow X$ satisfies condition (2) of the definition of $C_{n}$-forms, then there exists $Q_{n}: K_{n} \times X^{n} \rightarrow X$ such that $\left\{Q_{i}\right\}, i=1, \ldots$, $n$, is a $C_{n}$-form on $X$.

The main results on $C_{n}$-spaces relate the algebraic property given by the $C_{n}$-form to certain geometric properties of $X$. These results may be phrased in terms of quasifibrations. Hence, recall the following definition.

Definition 11. A continuous surjection $p: E \rightarrow B$ is called a quasifibration, q.f. for short, provided that

$$
p_{*}: \pi_{*}\left(E, p^{-1}(x), y\right) \rightarrow \pi_{*}(B, x)
$$

is an isomorphism for all $x$ in $B$ and $y$ in $p^{-1}(x)$.

Definition 12. A quasifibration $p: E \rightarrow B$ is called principal, provided that the fiber $X=p^{-1}(*)$ is a monoid which acts on $E$ in such a manner that (if $N: X \times E \rightarrow E$ denotes the action):

(1) $N(*, y)=y$,

(2) $N(X \times\{y\}) \subset p^{-1}(p(y))$,

(3) $N\left(m\left(x, x^{\prime}\right), y\right)=N\left(x, N\left(x^{\prime}, y\right)\right)$, and

(4) $N\left(x, x^{\prime}\right)=m\left(x, x^{\prime}\right)$,

for all $x, x^{\prime}$ in $X$ and all $y$ in $Y$. Here $m$ denotes the multiplication of $X$.

Definition 13. Let $X$ be a space. Then $(X)_{n}$, the (James) $n$-fold reduced product space of $X$, is obtained by identifying points of $X^{n}$ with each other if and only if they are the same when occurrences of the base point are disregarded. There is an obvious inclusion $(X)_{n} \subset(X)_{n+1}$ and the space obtained by taking the union of all $(X)_{n}$ under these inclusions (with the weak topology) is called $(X)_{\infty}$. (It is well known that $(X)_{\infty}$ is the homotopy type of $\Omega S X$, cf. [6], whenever $X$ is a connected CW-complex.)

MAIN THEOREM 14. Let $X$ be a countable $C W$-monoid. Then the following conditions are equivalent.

(1) $X$ admits a $C_{n}$-form.

(2) The Hopf construction for $X, p_{2}: E_{2} \rightarrow S X$, extends to a principal quasifibration $p: E_{n} \rightarrow B_{n}$, where $B_{n}$ is the homotopy type of $(S X)_{n}$.

(3) There is an n-homotopy-multiplicative map $d: \Omega(S X)_{n} \rightarrow X$ such if $j: X \rightarrow$ $\Omega(S X)_{n}$ is the usual inclusion given by $j(x)[t]=t \wedge x$ in $S X$, considered as a subspace of $(S X)_{n}$, then dj is homotopic to the identity map of $X$.

$\S 3$ will be devoted to the proof of this theorem, but we present here an outline of the proof. The implication (1) $\Rightarrow(2)$ will be proven by means of a direct construction in the Dold-Lashof vein.

The implication $(2) \Rightarrow(3)$ will be derived from the next two results. 
Lemma 15. If $p: E \rightarrow B$ is any q.f., there is a "connecting map" $d: \Omega B \rightarrow F$, where $F$ is the fiber. If $p$ extends the Hopf map $p_{2}$, then dj is homotopic to the identity map of $F$.

THEOREM 16. If $p: E \rightarrow B$ is any principal q.f., then the connecting map d: $\Omega B \rightarrow F$ is strongly homotopy-multiplicative.

Lemma 15 is standard, and the proof of Theorem 16 may be found in [3].

There is a well known concept of what is meant by a sub- $H$-space of an $H$-space being homotopy-commutative in the containing space, see [7]. In $\$ 3$ there will be given an analogous definition of a submonoid, $Y$, being $C_{n}$ in a containing monoid, $X$. The implication $(3) \Rightarrow(1)$ is based on the following lemma.

LEMMA 17. Let the inclusion $\Omega S X \subset \Omega(S X)_{n}$ be induced by the inclusion $S X \subset(S X)_{n}$. Then $\Omega S X$ is $C_{n}$ in $\Omega(S X)_{n}$.

An application of our Main Theorem is the following corollary.

Corollary 18. A countable $C W$-complex, $X$, is a $C_{\infty}$-space if and only if there is a strongly homotopy-multiplicative map $d: \Omega^{2} S^{2} X \rightarrow X$ such that dj is homotopic to the identity, where $j: X \rightarrow \Omega^{2} S^{2} X$ is given by the composition $X \rightarrow \Omega S X \rightarrow \Omega^{2} S^{2} X$.

REMARK 19. There is another characterization of $C_{n}$-spaces which, owing to the length of its proof, will merely be noted here, its development being reserved for a subsequent paper. It is as follows: $A$ countable $C W$-monoid, $X$, is a $C_{n}$-space if and only if there is a map a: $(S X)_{n} \rightarrow P_{n}(X)$ which extends the identity map $1: S X \rightarrow$ $S X=P_{1}(X)$. Here $P_{n}(X)$ is the $n$th projective space of $X$, as constructed in [1].

This characterization is significant in the following two respects. First, it extends the theorem of Stasheff, from [11], that a homotopy-associative $H$-space is homotopy-commutative if and only if the map $a:(S X)_{2} \rightarrow P_{2}(X)$ exists. Secondly, such a map $a:\left(S^{2}\right)_{n} \rightarrow P_{n}\left(S^{1}\right)$, obtained by other means, is exploited by Toda, p. 174 of [16], in his computations of homotopy groups of spheres. Thus the existence of his map seems to be related to the commutativity of $S^{1}$.

The idea of the proof of this characterization is to obtain a fiber-preserving map from the total space, $E_{n}$, of Theorem 14, to the total space of the Dold-Lashof construction, $E_{n}$.

It is a natural question to ask what the maps are in the category of $C_{n}$-spaces, i.e., what are the maps which sufficiently respect the $C_{n}$-forms. Just as in the category of $H$-spaces it is more useful to consider the $H$-maps rather than only the strict homomorphisms, the maps in the $C_{n}$-category will be taken to be those which preserve the structure up to homotopy.

In his work with $A_{n}$-spaces, Stasheff in [12] determines necessary and sufficient conditions to decide when the total space of a fibration induced from a path space fibration admits an $A_{n}$-form. His methods serve to motivate the following definitions and to provide models for the resulting theorems, which answer the analogous questions for $C_{n}$-forms. 
Definition 20. Let $\left(X,\left\{Q_{i}^{X}\right\}\right)$ and $\left(W,\left\{Q_{i}^{W}\right\}\right)$ be $C_{n}$-spaces. Then a map $f: X \rightarrow W$ is called a $C_{n}$-map, provided that $f$ is a homomorphism of monoids and that there exist maps

$$
D_{i}: I \times K_{i} \times X^{i} \rightarrow W, \quad i=2, \ldots, n,
$$

such that

$$
\begin{gathered}
D_{i}\left(0, \tau, x_{1}, \ldots, x_{i}\right)=Q_{i}^{W}\left(\tau, f\left(x_{1}\right), \ldots, f\left(x_{i}\right)\right) \\
D_{i}\left(1, \tau, x_{1}, \ldots, x_{i}\right)=f\left(Q_{i}^{X}\left(\tau, x_{1}, \ldots, x_{i}\right)\right) \\
D_{i}\left(t, V\left(A_{r}, B_{s}\right)[\rho, \sigma], x_{1}, \ldots, x_{i}\right) \\
=D_{r}\left(t, \rho, x_{A(1)}, \ldots, x_{A(r)}\right) \cdot D_{s}\left(t, \sigma, x_{B(1)}, \ldots, x_{B(s)}\right)
\end{gathered}
$$

and

$$
D_{i}\left(t, \tau, x_{1}, \ldots, x_{i}\right)=D_{i-1}\left(t, s_{j}(\tau), x_{1}, \ldots, \hat{x}_{j}, \ldots, x_{i}\right)
$$

if $x_{j}=e$, the identity of $X$.

If $X$ and $W$ are $C_{\infty}$-spaces, and the $D_{i}$ exist for all $i \geqq 1$, then $f$ is called a $C_{\infty}$-map. EXAMPLES.

(1) A homomorphism of abelian monoids is a $C_{\infty}$-map.

(2) Let $X$ and $Y$ be $H$-spaces and $f: X \rightarrow Y$ be an $H$-map. Then $f: \Omega X \rightarrow \Omega Y$ is a $C_{\infty}$-map.

The following theorem illustrates the use of $C_{n}$-maps by giving an extension theorem for $C_{n}$-forms.

THEOREM 21. Let $X$ and $W$ be countable $C W C_{n}$-spaces and $f: X \rightarrow W$ be a $C_{n}$-map. Let $p: Y \rightarrow X$ be the fibration induced by $f$ from the standard fibration $\pi: P W \rightarrow W$. Then $Y$ may be given the structure of a $C_{n}$-space in such a way that $p$ is a $C_{n}$-map.

Partial converses to this theorem may be easily obtained by making dimensionality restrictions on the homotopy and cohomology groups of the spaces involved in order to insure that certain obstructions vanish. The next two theorems, which are of a rather technical nature, illustrate this method.

THEOREM 22. Let $p: Y \rightarrow X$ be the fibration induced from $\pi: P W \rightarrow W$ by a map $f: X \rightarrow W$. Suppose that $X, Y$, and $W$ are $C_{n}$-spaces, that $p$ is a $C_{n}$-map, and that $f$ is a $C_{n-1}$-map. Further, suppose that there exist integers $p$ and $q, q \geqq p \geqq 2$ such that

(1) $\pi_{i}(X)=0$, for $i \leqq p-1$, and

(2) $\pi_{i}(W)=0$, for $i \leqq q$ and $i \geqq(n-1) p+q$.

Then $f$ is a $C_{n}$-map.

THEOREM 23. Let $\left(X,\left\{Q_{i}^{X}\right\}\right)$ be a $C_{n-1}$-space and let $\left(Y,\left\{Q_{i}^{Y}\right\}\right)$ be a $C_{n}$-space. Let $p: Y \rightarrow X$ be a fibration with fiber $F$ which is a $C_{n-1}$-map. Further, suppose that there exist integers $p$ and $q, q \geqq p \geqq 2$, such that

(1) $\pi_{i}(X)=0$, for $i \leqq p-1$ and $i \geqq(n-1)(p+1)+q$, and

(2) $\pi_{i}(F)=0$, for $i \leqq q-1$. 
Then there exist $Q_{n}^{K}: K_{n} \times X^{n} \rightarrow X$ such that

(a) $\left\{Q_{i}^{X}\right\}$ is a $C_{n}$-form on $X$, and

(b) $p$ is a $C_{n}$-map.

The proofs of the last three theorems will be outlined in §3. These theorems, together with Proposition 8 yield the following application, cf. [12, pp. 301-302].

TheOREM 24. A space with a two-stage Postnikov system admits a $C_{n}$-form if and only if its $k$-invariant is represented by a $C_{n}$-map.

3. Let us begin this section by considering Example 3 of the preceding section, namely that the space of loops on an $H$-space is a $C_{\infty}$-space. Its proof will follow from the proof of Lemma 17. We need the following definition.

Definition 25. Let $Y$ be a monoid, $X$ a space. A map $\psi: Z \rightarrow Y$ is called a $C_{n}$-commutativity of $Z$ into $Y$ if there are maps

$$
Q_{i}: K_{i} \times Z^{i} \rightarrow Y, \quad i=1, \ldots, n
$$

satisfying conditions (2) and (3) of the definition of $C_{n}$-form, but with condition (1) replaced by

(1) $Q_{1}: Z \rightarrow Y$ is just $\psi: Z \rightarrow Y$.

We now restate Lemma 17 .

Lemma 17. Let $\psi: \Omega X \rightarrow \Omega(X)_{n}$ be induced by the inclusion $X \rightarrow(X)_{n}$. Then $\psi$ is a $C_{n}$-commutativity of $\Omega X$ into $\Omega(X)_{n}$.

Proof. This proof is an extension of the familiar one showing that the loop-space of an $H$-space is homotopy-commutative. It is based on the fact that there are the two "multiplications" of $\Omega X$ in $\Omega(X)_{n}$. One is the loop additions, and the other is given by $\left(\lambda_{1}^{r_{1}}, \lambda_{2}^{r_{1}}\right) \rightarrow\left[\lambda_{1}^{r_{1}}, \lambda_{2}^{r_{2}}\right]$, where $\left[\lambda_{1}^{r_{1}}, \lambda_{2}^{r_{2}}\right]$ is the loop in $(X)_{n}$ of length $\max \left(r_{1}, r_{2}\right)$

$$
\begin{aligned}
& {\left[\lambda_{1}^{r_{1}}, \lambda_{2}^{r_{2}}\right](t)=\left[\lambda_{1}^{r_{1}}(t), \lambda_{2}^{r_{2}}(t)\right], \quad t=\min \left(r_{1}, r_{2}\right),} \\
& =\left[\lambda_{1}^{r_{1}}\left(r_{1}\right), \lambda_{2}^{r_{2}}(t)\right], \quad \text { if } r_{1}=\min \left(r_{1}, r_{2}\right), \\
& =\left[\lambda_{1}^{r_{1}}(t), \lambda_{2}^{r_{2}}\left(r_{2}\right)\right], \quad \text { if } r_{2}=\min \left(r_{1}, r_{2}\right) .
\end{aligned}
$$

Furthermore, $\left[\lambda_{1}^{r_{1}}, \ldots, \dot{\lambda}_{i}^{r_{i}}\right]$ is defined for $i \leqq n$ by iteration. Now assume inductively that $Q_{j}$ has been defined for $j<i$ in such a manner that

$$
Q_{i}\left(\tau, \lambda_{1}^{r_{1}}, \ldots, \lambda_{i}^{r_{i}}\right)=\left[\ldots, e^{a_{j}}+\lambda_{j}^{b_{j}}+e^{c_{j}}, \ldots\right],
$$

when given by requirement (2) of the definition. Here $\lambda_{j}^{b}$ denotes $\lambda_{j}^{r}$ reparametrized to the length $b_{j}$ and $e^{a_{i}}$ is the constant path at the basepoint of length $a_{i}$.

Then we may regard $K_{i}$ as the cone on its boundary and set

$$
Q_{i}\left(\tau \wedge s, \lambda_{1}^{r_{i}}, \ldots, \lambda_{i}^{r^{i}}\right)=\left[\ldots, e^{s a_{j}}+\lambda_{j}^{b_{j}+(1-s)\left(a_{j}+c_{j}\right)}+e^{s c_{j}}, \ldots\right],
$$

which accomplishes the definition of $Q_{i}$.

As a corollary, we have Example 3. 
COROllaRY 26. If $X$ is an $H$-space, then $\Omega X$ is a $C_{n}$-space for all $n$.

Proof. Let $(X)_{n} \stackrel{\oplus}{\rightarrow} X$ be given by multiplication, associating from the right. Then the composition

$$
K_{i} \times(\Omega X)^{i} \stackrel{Q_{i}}{\longrightarrow} \Omega(X)_{n} \stackrel{\Omega \phi}{\longrightarrow} \Omega X, \quad 1 \leqq i \leqq n
$$

is a $C_{n}$-form in $X$, where $Q_{i}$ is the map of the preceding lemma.

We now turn to the proof of Proposition 8. It will be accomplished by induction on $i$, the degree of the $C_{i}$ form, then by induction on the skeleta of $X$.

Proof of Proposition 8. Suppose $Q_{j}^{X}: K_{j} \times X^{j} \rightarrow X$ has been defined for $j<i$, in such a way that there is a homotopy, $H_{j}$, between $f \circ Q_{j}^{X}$ and $Q_{j}^{Y} \circ\left(1 \times f^{j}\right)$ satisfying the consistency hypothesis stated below. Our problem is to construct a $C_{i}$-form on $X$. Its value is already specified on $Q_{i}^{X}: \operatorname{Bd}\left(K_{i}\right) \times X^{i} \rightarrow X$.

It suffices to show that there is a homotopy $H_{i}: I \times \mathrm{Bd}\left(K_{i}\right) \times X^{i} \rightarrow Y$ between $f \circ Q_{i}^{X}$ and $Q_{i}^{Y} \circ\left(1 \times f^{i}\right)$. Then making use of the fact that $f$ induces isomorphisms of homotopy groups, $Q_{i}^{X}$ may be extended by induction on the skeleta of $K_{i} \times X^{i}$ in such a way that $H_{i}$ extends to become a homotopy between $f \circ Q_{i}^{X}$ and $Q_{i}^{Y} \circ\left(1 \times f^{i}\right)$ on all of $I \times K_{i} \times X^{i}$.

Such a homotopy exists on each top-dimensional face of $\mathrm{Bd}\left(K_{i}\right)$, but these homotopies may not coincide on the intersections of the faces. Specifically suppose $\sum_{j=1}^{k} r_{j}=i$ and faces of the form $K_{r_{1}+\cdots+r_{j}} \times K_{r_{j+1}+\cdots+r_{k}}$ intersect; $j=1, \ldots, k-1$. We may write their intersection as $K_{r_{1}} \times \cdots \times K_{r_{k}}$. Let $\left(\rho_{1}, \ldots, \rho_{k}\right)$ be a point in this intersection. Then for each $j$, we have a homotopy

$$
\begin{aligned}
f\left(Q_{1}^{X}\left(\rho_{1}, x_{A^{1}(1)}, \ldots, x_{A^{1}\left(r_{1}\right)}\right) \cdots \cdots Q_{r_{k}}^{X}\left(\rho_{k}, x_{A^{k}(1)}, \ldots\right)\right) \\
\quad=f\left(Q_{\alpha}^{X}\left(\rho_{\alpha}, x_{A^{\alpha}(1)}, \ldots, x_{A^{\alpha}(\alpha)}\right) \cdot Q_{\beta}^{X}\left(\rho_{\beta}, x_{A^{\beta}(1)}, \ldots\right)\right) \\
\sim f\left(Q_{\alpha}^{X}\left(\rho_{\alpha}, x_{A^{\alpha}(1)}, \ldots\right) \cdot f\left(Q_{\beta}^{X}\left(\rho_{\beta}, x_{A^{\beta}(1)}, \ldots\right)\right)\right. \\
\sim Q_{\alpha}^{Y}\left(\rho_{\alpha}, f\left(x_{A^{\alpha}(1)}\right), \ldots\right) \cdot Q_{\beta}^{Y}\left(\rho_{\beta}, f\left(x_{A^{\beta}(1)}\right), \ldots\right), \text { by } D_{\alpha} \times D_{\beta}, \\
\quad=Q_{r_{1}}^{Y}\left(\rho_{1}, f\left(x_{A^{1}(1)}\right), \ldots\right) \cdots \cdots Q_{r_{k}}^{Y}\left(\rho_{k}, f\left(x_{A^{k}(1)}\right), \ldots\right) .
\end{aligned}
$$

Here $\alpha=r_{1}+\cdots+r_{j}, \beta=r_{j+1}+\cdots+r_{k}$, and $\left(A^{\alpha}, A^{\beta}\right)$ is the $(\alpha, \beta)$ partition which refines to the $\left(r_{1}, \ldots, r_{k}\right)$ partition $\left(A^{1}, \ldots, A^{k}\right)$.

Then in order to piece together the homotopies, exactly what is required is that $f$ be a $k$-homotopy-multiplicative map.

Now let us prove Theorem 13.

Proof that (1) $\Rightarrow(2)$. We begin with $E_{0}=X$ and $a_{1}: X \rightarrow X$ the identity map. Let $Z_{i}=\left(K_{i} \times X \times X^{[i-1]}\right) \cup\left(\mathrm{Bd}\left(K_{i}\right) \times X^{i}\right) \subset K_{i} \times X^{i}$. Here $X^{[i-1]}$ denotes the subspace of $X^{i-1}$ consisting of those points at least one of whose coordinates is the basepoint. Then $p_{i}: E_{i} \rightarrow B_{i}, i \leqq n$, are constructed inductively as follows: Let

$$
a_{i}:\left(K_{i} \times X^{i}, Z_{i}\right) \rightarrow\left(E_{i-1}, E_{i-2}\right)
$$

be a relative homeomorphism, in which $a_{i} \mid Z_{i}$ is given by 
(1) if $x_{j}=e$, the identity of $X$, then

$$
a_{i}\left(\tau, x, x_{1}, \ldots, x_{i-1}\right)=a_{i-1}\left(s_{j}(\tau), x, x_{1}, \ldots, \hat{x}_{j}, \ldots, x_{i-1}\right),
$$

(2) if $\left(A_{r}, B_{s}\right)$ is a partition of $i$, and $i \in A_{r}$, then

$$
a_{i}\left(V\left(a_{r}, B_{s}\right)[\rho, \sigma], x, x_{1}, \ldots, x_{i-1}\right)=a_{r}\left(\rho, x, x_{A^{\prime}(1)}, \ldots, x_{A^{\prime}(r-1)}\right),
$$

where $A_{r-1}^{\prime}=A_{r}-(i)$, and

(3) if $\left(A_{r}, B_{s}\right)$ is a partition of $i$, and $i \in B_{s}$, then

$$
\begin{aligned}
a_{i}\left(V\left(A_{r}, B_{s}\right)[\rho, \sigma],\right. & \left.x, x_{1}, \ldots, x_{i-1}\right) \\
& =a_{s}\left(\sigma, x \cdot Q_{r}\left(\rho, x_{A(1)}, \ldots, x_{A(r)}\right), x_{B^{\prime}(1)}, \ldots, x_{B^{\prime}(s-1)}\right)
\end{aligned}
$$

where $B_{s-1}^{\prime}=B_{s}-(i)$.

Let $B_{0}$ be a point. Then let $b_{i}:\left(K_{i} \times X^{i-1}, W_{i}\right) \rightarrow\left(B_{i-1}, B_{i-2}\right)$ be a relative homeomorphism, where

$$
W_{i}=\left(K_{i} \times X^{[i-1]}\right) \cup\left(\mathrm{Bd}\left(K_{i}\right) \times X^{i-1}\right)
$$

and $b_{i} \mid W_{i}$ is defined by formulas (1), (2), and (3) with $x$ omitted. Now define $p_{i}: E_{i} \rightarrow B_{i}$ as being induced from the projection $K_{i} \times X \times X^{i-1} \rightarrow K_{i} \times(e) \times X^{i-1}$ $=K_{i} \times X^{i-1}$.

The action $N_{i}: X \times E_{i} \rightarrow E_{i}$ is defined by

$$
N_{i}\left(z, \alpha_{i}\left(\tau, x, x_{i}, \ldots, x_{i-1}\right)\right)=\alpha_{i}\left(\tau, z x, x_{1}, \ldots, x_{i-1}\right) .
$$

First we verify that $B_{n}$ is homotopic to $(S X)_{n}$. Recall that $(S X)_{n}$ may be formed by relative homeomorphisms:

$$
c_{i}:\left(I^{i} \times X^{i},\left(\operatorname{Bd}\left(I^{i}\right) \times X^{i}\right) \cup\left(I^{i} \times X^{[i]}\right)\right) \rightarrow\left((S X)_{i},(S X)_{i-1}\right), \quad i=1, \ldots, n,
$$

given by

$$
\begin{aligned}
c_{i}\left(t_{1}, \ldots, t_{i}, x_{1}, \ldots, x_{i}\right)=c_{i-1}\left(t_{1}, \ldots, \hat{t}_{k}, \ldots, t_{i}, x_{1}, \ldots, \hat{x}_{k}, \ldots, x_{i}\right) \\
\text { if } t_{i}=0 \text { or } 1, \text { or if } x_{k}=e .
\end{aligned}
$$

Now consider the map $g_{i}: K_{i} \rightarrow I^{i-1}$ given on the vertices of $K_{i}$ as follows. Let $\left(r_{1}, \ldots, r_{i}\right)$ be a vertex of $K_{i}$ given by an element $\gamma \in S(i)$ by $\left(r_{1}, \ldots, r_{i}\right)=\gamma(1, \ldots, i)$. Then set $g_{i}\left(r_{1}, \ldots, r_{i}\right)=\left(t_{2}, \ldots, t_{i}\right) \in I^{i-1}$ where

$$
\begin{array}{rlrl}
t_{k} & =0, & \text { if } \gamma(1)<\gamma(i), \\
& =1, & & \text { if } \gamma(i)<\gamma(1) .
\end{array}
$$

It is easily verified that the image of the set of vertices of a face of $K_{i}$ is the set of vertices of a face of $I^{i-1}$. Hence $g_{i}$ can be extended by linearity to map $\operatorname{Bd}\left(K_{i}\right)$ onto Bd $\left(I^{i-1}\right)$. Furthermore, it is clear how to deform $g_{i}$ into a homeomorphism. Now extend $g_{\mathfrak{l}}$ to 'map $g_{\mathfrak{i}}: K_{\mathfrak{i}} \rightarrow I^{i}$ in such a way that $g_{\mathfrak{i}}$ restricted to Int $\left(K_{\mathfrak{i}}\right)$ is a homeomorphism. Let $\psi_{1}: B_{1} \rightarrow S X$ be the identity map. We shall define inductively 
maps $\psi_{i}: B_{i} \rightarrow(S X)_{i}$ such that $\psi_{i} \mid B_{i-1}=\psi_{i-1}$. Suppose we have done this for $j<i$ in such a way that the diagram

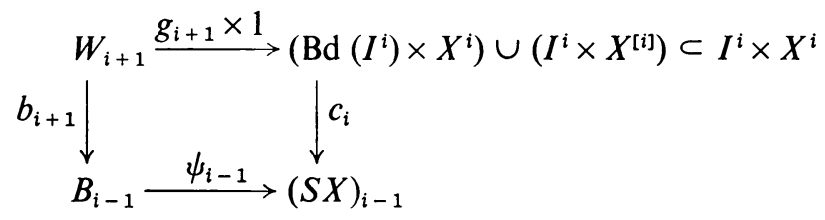

is commutative and such that $\psi_{i-1}$ is a homotopy equivalence. Then $\psi_{i-1}$ can be extended to a homotopy equivalence, $\psi_{i}: B_{i} \rightarrow(S X)_{i}$. The fact that this $\psi_{i}$ satisfies the inductive hypothesis follows from the definitions of $g_{i+2}, b_{i+2}$, and $c_{i+1}$.

To complete the implication $(1) \Rightarrow(2)$, it remains to show that $p_{i}: E_{i} \rightarrow B_{i}$ is a quasifibration. The method of proof is due to Dold and Thom [2]. The base, $B_{i}$, may be expressed as the union of two open subsets, $U$ and $W$, such that $p_{i}$ is a q.f. over each of $U, W$, and $U \cap W$. The details are similar to those in the proof of Theorem 19 on p. 286 of [12] and hence will be omitted.

As mentioned in $\$ 2$, the implication $(2) \Rightarrow(3)$ follows immediately from Lemma 15 and Theorem 16. We now consider the implication $(3) \Rightarrow(1)$.

Proof that $(3) \Rightarrow(1)$. By Lemma 17, there are maps $\hat{Q}_{i}: K_{i} \times(\Omega S X)^{i} \rightarrow \Omega(S X)_{n}$, $i=1, \ldots, n$, realizing the inclusion $\Omega S X \subset \Omega(S X)_{i}$ as a $C_{n}$-commutativity of $\Omega S X$ into $\Omega(S X)_{i}$. Composition with the canonical inclusion of $X$ into $\Omega S X$ yields a $C_{n}$-commutativity of $X$ into $\Omega(S X)_{n}$ realized by maps $\bar{Q}_{i}: K_{i} \times X^{i} \rightarrow \Omega(S X)_{i}$. Our first attempt to define a $C_{n}$-form on $X$ would be to define $Q_{i}: K_{i} \times X^{i} \rightarrow X$ to be the composition

$$
K_{i} \times X^{i} \stackrel{\bar{Q}_{i}}{\longrightarrow} \Omega(S X)_{i} \stackrel{d}{\longrightarrow} X .
$$

This attempt would succeed if $d$ were actually a homomorphism. Since $d$ is only an $n$-homotopy-multiplicative map, however, this definition does not work. Instead, the $Q_{i}$ have to be defined inductively, using a procedure similar to that of the proof of Proposition 9. (An alternative approach would be to replace $d$ by an equivalent map which is a homomorphism.) With this modification, the construction of the $C_{n}$-form on $X$ can be completed. This finishes the proof of Theorem 14.

Now we turn to the results on $C_{n}$-maps and on $C_{n}$-forms in induced fibrations. We begin by remarking that the claim in Example 2 that the loop map of an $H$-map is a $C_{\infty}$-map is easily verified using the same homotopies which were used in constructing the $C_{\infty}$-forms on $\Omega X$ and $\Omega Y$. We now proceed to the next theorem.

Proof of Theorem 21. Let $m^{X}, Q_{i}^{X}, m^{w}, Q_{i}^{W}$, be the multiplications and $C_{i}$-forms on $X$ and $W$, respectively. We are given maps

Let

$$
D_{i}: I \times K_{i} \times X^{i} \rightarrow W, \quad 2 \leqq i \leqq n .
$$

$$
\hat{D}_{i}: K_{i} \times X^{i} \rightarrow W^{I} \times R \quad(R \text { the reals })
$$

be the adjoint of $D_{i}$, deformed to make it basepoint-preserving, keeping the deformation through paths satisfying the boundary conditions of Definition 21 . 
We may use the multiplication and $C_{i}$-forms on $W$ to induce laws of composition, $\hat{m}^{W}$ and $\hat{Q}^{W}$, on $W^{I}$, in similar fashion to that in the proof of Lemma 17.

The multiplication $m^{Y}$, on $Y$ and the $C_{i}$-forms, $Q_{i}^{Y}$, on $Y$ may be given as follows:

$$
m^{Y}\left(\left(x_{1}, a_{1}\right),\left(x_{2}, a_{2}\right)\right)=\left(m^{X}\left(x_{1}, x_{2}\right), \hat{m}^{W}\left(a_{1}, a_{2}\right)\right)
$$

and

$$
\begin{aligned}
& Q_{i}^{Y}\left(\tau,\left(x_{1}, a_{1}\right), \ldots,\left(x_{i}, a_{i}\right)\right) \\
& \quad=\left(Q_{i}^{X}\left(\tau, x_{1}, \ldots, x_{i}\right), \hat{Q}_{W}^{i}\left(\tau, a_{1}, \ldots, a_{i}\right)+\hat{D}_{i}\left(\tau, x_{1}, \ldots, x_{i}\right)\right) .
\end{aligned}
$$

Here $\tau \in K_{i}, x_{i}, \ldots, x_{i} \in X$, and $a_{1}, \ldots, a_{i} \in P W$.

The verification that these definitions satisfy the requirements is straightforward.

We now turn to the proofs of Theorems 22 and 23.

Proof of Theorem 22. What is required is a map $D_{n}: I \times K_{n} \times X^{n} \rightarrow W$ which satisfies the boundary conditions as given in Definition 20. These conditions already determine $D_{n}$ on the subset Bd $\left(I \times K_{n}\right) \times X^{n} \cup I \times K_{n} \times X^{[n]}$.

The proof that the obstructions to extending $D_{n}$ to the rest of $I \times K_{n} \times X^{n}$ are zero uses the same methods as those in Proposition 10.5 on p. 309 of [12] and thus will be omitted. The proof of Theorem 23 uses the same techniques as Theorem 22 in locating the obstructions to the construction of $Q_{n}$ in trivial cohomology groups, and consequently we again refer to Stasheff's work in [12] for the methods of this proof.

\section{REFERENCES}

1. A. Dold and R. Lashof, Principal quasifibrations and fibre homotopy equivalence of bundles, Illinois J. Math. 3 (1959), 285-305.

2. A Dold and R. Thom, Quasifaserungen and unendliche symmetrische Produkte, Ann. of Math. (2) 67 (1958), 239-281.

3. B. Drachman, Doctoral thesis, Brown University, Providence, R. I., 1966.

4. H. Hopf, Über die Abbildungen von Sphären auf Sphären niedriger Dimension, Fund. Math. 25 (1935), 427-440.

5. I. M. James, On H-spaces and their homotopy groups, Quart. J. Math. Oxford Ser. (2) 11 (1960), 161-179.

6. - Reduced product spaces, Ann. of Math. 62 (1955), 170-197.

7. I. M. James and E. Thomas, Which Lie groups are homotopy-abelian?, Proc. Nat. Acad. Sci. U.S.A. 45 (1959), 737-740.

8. R. J. Milgram, Iterated loop spaces, Ann. of Math (2) 84 (1966), 386-403.

9. G. J. Porter, Higher order Whitehead products, Topology 3 (1965), 123-135.

10. E. Spanier and J. H. Whitehead, On fiber spaces in which the fiber is contractible, Comment. Math. Helv. 29 (1955), 1-8.

11. J. Stasheff, On homotopy Abelian H-spaces, Proc. Cambridge Philos. Soc. 57 (1961), 734-745.

12. - Homotopy-associativity of H-spaces. I, II, Trans. Amer. Math. Soc. 108 (1963), 275-292; 293-312.

13. M. Sugawara, On a condition that a space is an H-space, Math. J. Okayama Univ. 6 (1957), 109-129.

14. — , A condition that a space is group-like, Math. J. Okayama Univ. 7 (1957), 123-149. 
15. - On the homotopy-commutativity of groups and loop spaces, Mem. Coll. Sci. Univ. Kyoto Ser. A 33 (1960/61), 257-269.

16. H. Toda, Composition methods in homotopy groups of spheres, Ann. of Math. Studies No. 49, Princeton Univ. Press, Princeton, N. J., 1961.

17. F. D. Williams, A geometric condition that an H-space be homotopy-commutative, mimeographed, New Mexico State University, 1966.

New Mexico State University,

las Cruces, New Mexico 